\title{
Excellent Interim Treatment Response with Polatuzumab Vedotin
}

\section{Polatuzumab Vedotin ile Çok lyi Ara Tedavi Yanıtı}

\author{
(D) Özgür Mehtap1, (D Gözde Dağlıöz Görür2, (D) Serkan Ünal11, (D) Ayfer Gedük1 \\ ${ }^{1}$ Kocaeli University Facuty of Medicine, Department of Hematology, Kocaeli, Turkey \\ ${ }^{2}$ Kocaeli University Facuty of Medicine, Department of Nuclear Medicine, Kocaeli, Turkey
}
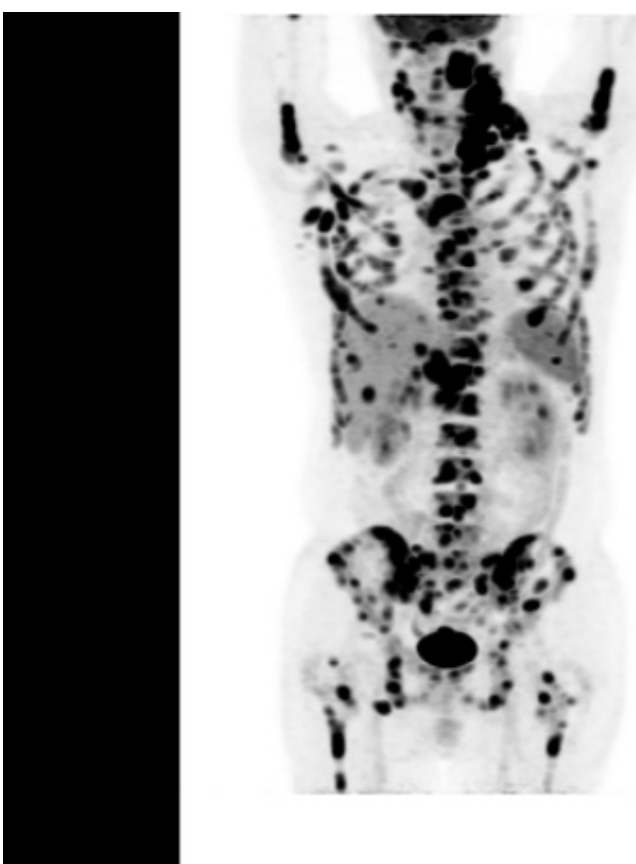

Figure 1. PET-CT imaging demonstrated widespread FDG uptake.
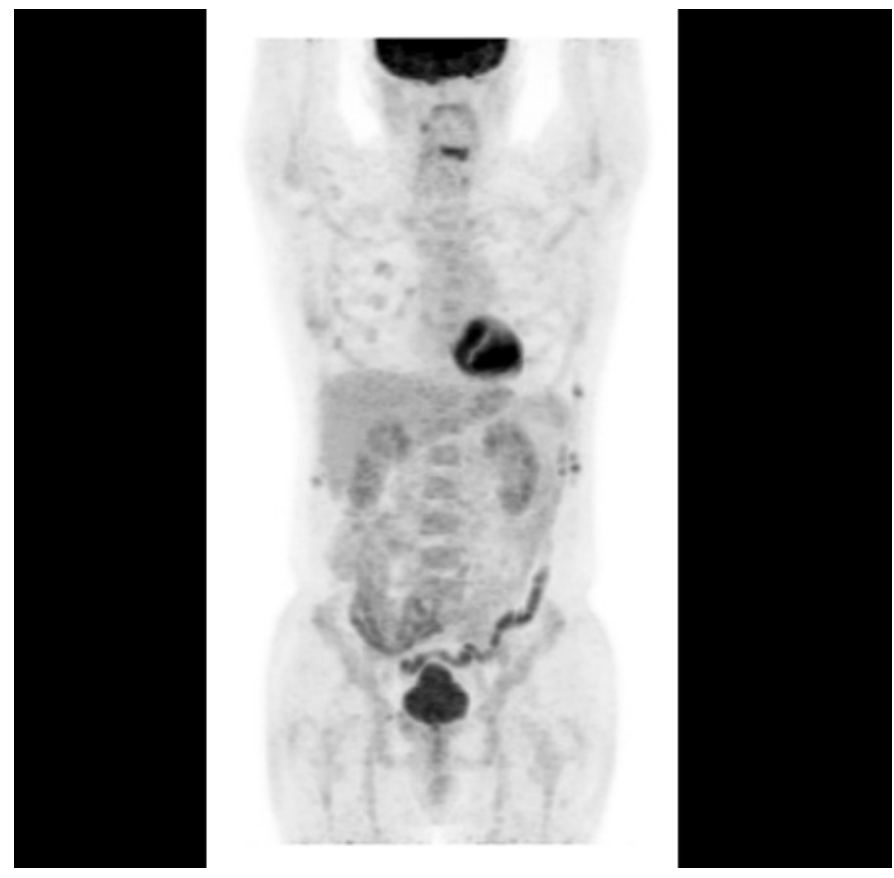

Figure 2. After three cycles of polatuzumab-rituximabbendamustine, PET-CT scanning demonstrated the disappearance of all FDG uptake except that in the costal bones.
A 57-year-old male presented with cervical mass and B symptoms for the last 2 months. The patient had a history of stage II diffuse large B-cell lymphoma (DLBCL), with complete response after six cycles of rituximab-cyclophosphamidedoxorubicin-vincristine-prednisone (RCHOP) chemotherapy in 2010. At the time of diagnosis, his International Prognostic Index score was low-intermediate. Three years after RCHOP treatment, the patient suffered from a cervical mass again.
Excisional lymph node biopsy confirmed relapse of DLBCL. After three cycles of rituximab-iphosphamide-carboplatin-etoposide (R-ICE) chemotherapy, the patient achieved a second complete response and underwent autologous stem cell transplantation. Five years after the transplantation, the disease relapsed again. Excisional lymph node biopsy revealed a relapse of the nongerminal type of DLBCL. The patient presented with the signs, symptoms, and laboratory findings of tumor lysis syndrome and 
paraneoplastic hypercalcemia. PET-CT imaging demonstrated widespread FDG uptake in the skeletal system (SUV max $_{2}$ 23.5), liver $\left(\right.$ SUV $_{\max }$ : 16.2), mediastinal lymph nodes (SUV ${ }_{\max }:$ 7.4), abdominal lymph nodes (SUV max $_{2}$ 26.6), cervical lymph nodes (SUV $_{\max }$ : 25.8), and left maxillary sinus (SUV $\left.{ }_{\max }: 29.5\right)$ (Figure 1). The patient received six cycles of polatuzumab vedotin (1.8 $\mathrm{mg} / \mathrm{kg} /$ day), rituximab (375 mg/m²/day), and bendamustine (90 $\mathrm{mg} / \mathrm{m}^{2} /$ day) every 21 days. After three cycles of polatuzumabrituximab-bendamustine, the patient was evaluated again by PET-CT scan, which demonstrated the disappearance of all FDG uptake except that in the costal bones, which had decreased significantly (SUV ${ }_{\max }: 23.5$ to 5.1) (Figure 2). This new drug can be effective and promising in this difficult-to-manage group of patients.

Keywords: Polatuzumab, DLBCL, PPET-CT, Image

Anahtar Sözcükler: Polatuzumab, DLBCL, PPET-BT, Görüntü

Conflict of Interest: No conflict of interest was declared by the authors.

Financial Disclosure: The authors declared that this study received no financial support. 